\title{
Does pregnancy increase the risk of multiple sclerosis? ${ }^{1}$
}

\author{
URI LEIBOWITZ, AARON ANTONOVSKY, RACHEL KATS, \\ AND MILTON ALTER
}

\author{
From the Department of Neurology, Hadassah University Hospital, Jerusalem, Israel, \\ and the Israel Institute of Applied Social Research, Jerusalem, Israel
}

The association of pregnancy and onset or relapse of multiple sclerosis is well known to every clinician and raises practical problems in clinical work. The problem of the relationship between multiple sclerosis and pregnancy and childbirth had been the subject of several investigations.

Early investigators (Beck, 1913) considered pregnancy so detrimental to patients with multiple sclerosis that therapeutic abortion was recommended. However, termination of pregnancy did not guarantee remission (von Hoesslin, 1934). Millar, Allison, Cheeseman, and Merrett (1959), in a careful analysis of the subject, concluded that the exacerbation rate of multiple sclerosis among women who had been pregnant was increased as compared with women who had not been pregnant, and Schapira, Poskanzer, Newell, and Miller (1966) also found that the frequency of relapse among women with multiple sclerosis who had been pregnant was somewhat higher than in non-pregnant patients.

In contrast to the above studies, there are others which purport to have found no detrimental effect of pregnancy upon the course of the disease (Müller, 1949; Tillman, 1950; McAlpine and Compston, 1952). Kurland (1952) pointed out that the childbearing period corresponds to the ages during which multiple sclerosis is active, namely, 15 to 45 . If a woman were pregnant only three times then she would spend about 36 months in a pregnant or post-partum state and chance association with the onset or exacerbation of multiple sclerosis of about the extent reported would be possible.

If pregnancy and delivery had an aetiological role in the pathogenesis of multiple sclerosis, then a comparison of the obstetrical histories of patients with multiple sclerosis and controls before the onset of illness might be of interest. The present study uses this approach, comparing the obstetrical history of

${ }^{1}$ This study was supported by grant no. 4X5111, Office of International Research, National Institutes of Health, Bethesda, Maryland 20014, U.S.A. patients with multiple sclerosis and controls in Israel before the onset of illness.

\section{MATERIAL AND METHOD}

In 1961, an intensive country-wide survey of multiple sclerosis in Israel was completed (Alter, Halpern, Kurland, Bornstein, Leibowitz, and Silberstein, 1962) and disclosed a total of 282 patients with the disease living in Israel as at 1 January 1960. In 1963, an effort was again made to contact the patients in order to carry out a study of their exposure to a variety of factors of possible aetiological importance. It was possible to locate and interview 241 patients from the original group of 282 identified in the nation-wide survey.

The controls were chosen by a cluster random sampling procedure from the 1961 population registry of the three major population centres of Israel. The controls selected were individually matched with patients on the basis of age, sex, and region of birth in a ratio of four controls to one patient.

In order to compare the same time period for patients and controls, an 'age at onset' was assigned to each control which was the same as the patient's with whom the control was matched. All questions were asked about events, habits, and patterns of living before the actual age at onset for patients and the assigned age at onset for controls.

The questionnaire formulated for the interview elicited not only obstetrical information but also data about many other events and experiences which had occurred before the age at onset. The method and results of that study have been reported elsewhere (Antonovsky, Leibowitz, Smith, Medalie, Balogh, Kats, Halpern, and Alter, 1965), and only the obstetrical information will be considered in this paper. The relevant questions pertained to number of pregnancies, abortions, live births, and stillbirths, and the percentage of patients and controls who reported having these conditions before the age at onset was calculated.

In 1965 a follow-up study was repeated in Israel. A questionnaire was designed to explore the areas in which the 1963 survey had revealed significant differences between patients and controls, but omitting those items on which there was no significant difference between patients and controls in the 1963 survey. Of the original 
patient population, 208 were found and interviewed. In addition, another 13 patients diagnosed after 1 January 1960 in the Department of Neurology of the Hadassah University Hospital in Jerusalem were added to bring the total number of patients in the 1965 study to 221. The 1965 control sample was selected by a stratified random sampling technique. This time only two controls were taken for each patient, individually matched by age, sex, and region of birth, similar to the method used in 1963. Details of the 1965 survey are given elsewhere (Antonovsky, Leibowitz, Medalie, Smith, Halpern, and Alter, 1967).

According to the method used in the study, questions about the number of pregnancies, deliveries, and abortions were not included in the 1965 questionnaire. However, in 1965 respondents were asked how old they were when they had iast become pregnant before the age at onset. The responses were then grouped by time intervals between the last pregnancy and the age at onset.

\section{RESULTS}

In 1963, a total of 131 female patients and 523 female controls was interviewed. Of these, $69 \%$ of the patients and $64 \%$ of the controls reported having been pregnant at least once before the age at onset. Table I shows the proportion of those who reported deliveries and abortions. There are no significant differences between patients and controls on any item.

\section{TABLE I}

PERCENTAGE OF RESPONDENTS WITH DELIVERIES AND ABORTIONS BEFORE THE AGE AT ONSET (1963 STUDY)

\begin{tabular}{lcc} 
& \multicolumn{1}{l}{ Percentage } \\
\cline { 2 - 3 } & Patients & Controls \\
\hline Never pregnant & 28 & 33 \\
Pregnant, no live births & 5 & 7 \\
Live births and stillbirths and abortions & 29 & 24 \\
Only live births & 35 & 33 \\
No answer (including respondents whose & 3 & 3 \\
$\quad$ age at onset was 15 or less) & 100 & 100 \\
Total & 131 & 523 \\
Number of cases &
\end{tabular}

In 1965 , a total of 122 female patients and 244 controls was interviewed. Of these, $60 \%$ of the patients and $56 \%$ of the controls reported having been pregnant at least once before the age at onset. Table II shows the time intervals between the last pregnancy and the age at onset. Among the patients, $23 \%$ were last pregnant two years or less before the age at onset, and $37 \%$ were last pregnant three or more years before it. Among the controls the percentages were 21 and 35 , respectively. However, when a division was made between one and two years before the age at onset, there was a significant difference: $16 \%$ of the patients and only $8 \%$ of the controls became pregnant less than one year before
T A BLE II

INTERVAL BETWEEN LAST PREGNANCY AND AGE AT ONSET (1965 STUDY)

\begin{tabular}{lcc} 
& \multicolumn{2}{l}{ Percentage } \\
\cline { 2 - 3 } & Patients & Controls \\
\hline $\begin{array}{l}\text { Never pregnant before age at onset } \\
\text { Became pregnant less than a year before } \\
\quad \text { age at onset }\end{array}$ & 34 & 38 \\
$\begin{array}{l}\text { Became pregnant 1-2 years before age at } \\
\text { onset }\end{array}$ & 7 & 8 \\
$\begin{array}{l}\text { Became pregnant 3-5 years before age at } \\
\text { onset }\end{array}$ & 14 & 13 \\
$\begin{array}{l}\text { Became pregnant more than 5 years before } \\
\text { age at onset }\end{array}$ & 23 & 13 \\
$\begin{array}{l}\text { No answer (including respondents whose } \\
\text { age at onset was 15 or less) }\end{array}$ & 6 & 22 \\
$\begin{array}{l}\text { Total } \\
\text { Number of cases }\end{array}$ & 100 & 6 \\
\end{tabular}

the age at onset $\left(\chi^{2}=5.7 ; P<0.02\right)$, while $7 \%$ of the patients and $13 \%$ of the controls last became pregnant one to two years before the age of onset.

One difference between the 1963 and the 1965 patient groups should be noted. In $1965,34 \%$ of the patients reported that they had never been pregnant before the age at onset, compared with $28 \%$ in 1963 . This may be due to the fact that the 1965 group had a somewhat younger age at onset than the 1963 group. The 1965 series did not include a number of women who died or could not be reached after 1963 . These were mostly older patients, many of whom experienced the onset of multiple sclerosis later. Also, the 13 patients who were added in 1965 changed the sample somewhat.

\section{DISCUSSION}

The present results indicate that there was no significant difference between patients and controls in the proportion of respondents who had been pregnant before the age at onset, and on the pattern of live births, stillbirths, and abortions. There was, however, a difference in the time interval from the last pregnancy to the age at onset. Significantly more patients than controls had become pregnant less than one year before the age at onset. On the other hand, more controls than patients reported pregnancy one to two years before the age at onset. There was no difference in the percentage reporting a last pregnancy three years or more before the age at onset.

The results do not support the notion that pregnancy and delivery have a direct relationship with the aetiology of multiple sclerosis, since the obstetrical history of patients and controls before the age at onset was similar. However, the time interval between the last pregnancy and the age at onset was different, suggesting that pregnancy may 
have a 'precipitating' effect. Women who were about to develop multiple sclerosis during the following two years tended to show clinical symptoms earlier if they became pregnant. Pregnancy may cause the illness to become manifest one year earlier than it would have been otherwise. If the onset of illness were due in three or more years, pregnancy had no effect on the time of appearance of clinical symptoms.

It may be postulated that in the two years before the onset of clinical manifestations of multiple sclerosis the patient is in a 'premorbid' state, during which pregnancy may have a precipitating effect and cause the clinical symptoms to become manifest earlier. Pregnancy three or more years before onset has no such effect.

A distinction must, however, be drawn between a 'precipitating' factor in multiple sclerosis and one which is of aetiological significance. Therefore, it should not be assumed from the data in this report that pregnancy is an aetiological agent. In a woman who was 'incubating' multiple sclerosis or in the 'premorbid' state, a variety of factors, including pregnancy, might cause the disease to become manifest. Our results suggest that this interval is not longer than two years as there was no excess of pregnancies among patients in the period three or more years before onset. The two years before the onset might be 'critical' for the patients, in that during this period they are susceptible to the influence of various factors which may precipitate the appearance of neurological symptoms. Perhaps factors other than pregnancy might have a similar influence, and disclosing these may shed some light on the causes and mechanisms of onset and relapse in multiple sclerosis.

An observation similar to the present one was reported by Millar et al. (1959) and Millar (1961) concerning relapses in patients who already had multiple sclerosis when pregnant. They found an increase in the exacerbation rate in the three post-partum months rather than during the nine months of pregnancy itself. Despite the increased exacerbation rate among the pregnant women, their disability was not increased compared with patients who had not been pregnant. This finding suggested that pregnancy tends to bring forward attacks which might have occurred anyhow.

Another analysis of the effect of pregnancy upon the course of multiple sclerosis has been reported by Schapira et al. (1966), who also found that the three post-partum months were particularly hazardous, and the overall frequency of relapse among women with multiple sclerosis who had been pregnant was higher than in non-pregnant patients. However, the increase amounted to an average of only 0.034 exacerbations per year.
In view of the importance of this question and the conflicting opinions in the literature, further study is warranted. However, the question is not an easy one to study since it is not clear what aspect of pregnancy or the puerperium may be of importance with respect to multiple sclerosis. For example, is the alleged effect related to hormonal changes, cerebral oedema, fluid and electrolyte balance, vascular changes, the disruption of the new mother's normal sleeping patterns and the attendant fatigue, or an anaesthetic used at the time of delivery.

The results of the present study should encourage investigations into the specific aspects of pregnancy or factors and events related to pregnancy as possible 'precipitating' agents. Attention should be focused on the two years before the onset of multiple sclerosis as the 'critical' period during which the patient is susceptible to the effect of such factors.

\section{SUMMARY}

Obstetrical histories before the onset of illness were obtained form a national series of patients with multiple sclerosis and from controls. No significant differences were found between patients and controls in the number of pregnancies, deliveries, and abortions. However, a significantly higher percentage of the patients reported a pregnancy in the year before onset, while relatively more controls had been pregnant one to two years before the age at onset. The percentage reporting pregnancies three or more years before onset was not significantly different in patients and controls. The results suggest that pregnancy may, on occasion, 'precipitate' the onset of multiple sclerosis. The hazardous period is during the last two years before the onset of clinical symptoms and it may correspond to an 'incubation' period or a 'premorbid state' during which a variety of factors, including pregnancy, may precipitate demyelination of the central nervous system.

\section{REFERENCES}

Alter, M., Halpern, L., Kurland, L. T., Bernstein, B., Leibowitz, U., and Silberstein, J. (1962). Multiple sclerosis in Israel. Prevalence among immigrants and native inhabitants. Arch. Neurol. (Chic.), 7, 253-263.

Antonovsky, A., Leibowitz, U., Medalie, J. M., Smith, H. A., Halpern, L., and Alter, M. (1967). Reappraisal of possible etiologic factors in multiple sclerosis. Amer. J. Publ. Hlth (in press).

- - - Smith H. A., Medalie, J. M., Balogh, M., Kats, R., Halpern, L., and Alter, M. (1965). Epidemiologic study of multiple sclerosis in Israel. I. An overall review of methods and findings. Arch. Neurol. (Chic.), 13, 183-193.

Beck, R. (1913). Multiplen Sklerose, Schwangerschaft und Geburt. Dtsch. Z. Nervenheilk, 46, 127-145.

von Hoesslin, R. (1934). Uber Multiplen Sklerose: Exogene Ätiologie, Pathogenese und Verlauf. Lehmann, Munich. 
Kurland, L. T. (1952). Epidemiologic characteristics of multiple sclerosis. Amer. J. Med., 12, 561-571.

McAlpine, D., and Compston, N. (1952). Some aspects of the natural history of disseminated sclerosis. Quart. J. Med., 21, 135-167.

Millar, J. H. D. (1961). The influence of pregnancy on disseminated sclerosis. Proc. roy. Soc. Med., 54, 4-7.

_-, Allison, R. S., Cheeseman, E. A., and Merrett, J. D. (1959).
Pregnancy as a factor influencing relapse in disseminated sclerosis. Brain, 82, 417-426.

Müller, R. (1949). Studies on disseminated sclerosis. Acta med. scand., 133, suppl. 222.

Schapira, K., Poskanzer, D. C., Newell, D. S., and Miller, H. (1966). Marriage, pregnancy, and multiple sclerosis. Brain, 89, 419-428. Tillman, A. J. B. (1950). The effect of pregnancy on multiple sclerosis and its management. Res. Publ. Ass. Res. nerv. ment. Dis., 28, 548-582. 